\title{
A Novel Equivalent Wiener-Hopf Equation with TDL coefficient in Lattice Structure
}

\author{
Ju-Phil Cho, Bong-Man Ahn, and Jee-Won Hwang, Member, KIMICS
}

\begin{abstract}
In this paper, we propose an equivalent Wiener-Hopf equation. The proposed algorithm can obtain the weight vector of a TDL(tapped-delay-line) filter and the error simultaneously if the inputs are orthogonal to each other. The equivalent Wiener-Hopf equation was analyzed theoretically based on the MMSE(minimum mean square error) method. The results present that the proposed algorithm is equivalent to original Wiener-Hopf equation. The new algorithm was applied into the identification of an unknown system for evaluating the performance of the proposed method. We compared the Wiener-Hopf solution with the equivalent Wiener-Hopf solution. The simulation results were similar to those obtained in the theoretical analysis.

In conclusion, our method can find the coefficient of the TDL (tapped-delay-line) filter where a lattice filter is used, and also when the process of Gram-Schmidt orthogonalization is used. Furthermore, a new cost function is suggested which may facilitate research in the adaptive signal processing area.
\end{abstract}

Index Terms - Wiener-Hopf equation, equivalent WienerHopf equation, MMSE(minimum mean square error), Gram-Schmidt orthogonalization, lattice filter, coefficient.

\section{INTRODUCTION}

THE Wiener-Hopf equation is a core algorithm for developing new adaptation algorithms [1]-[3]. A lattice filter has generally been utilized for the modeling of linear time-varying systems[4],[5]. However, lattice filters have various constraints in these applications, since the coefficients of the lattice filter are not TDL filter coefficients[6],[7].

Generally, the lattice filter constitutes a prediction stage and a joint process estimation stage. The prediction stage produces reflection coefficients and the joint process estimation stage produce regression coefficients, respectively[1]-[3]. These can be calculated by using the Wiener-Hopf equation. Therefore, the weakness of the lattice filter is that the coefficient of the TDL filter cannot

Manuscript received June 20, 2011; revised July 14, 2011; accepted July 25, 2011.

Ju-Phil Cho is with the Department of Radio-communication Eng., KUNSAN National Unversity, JEONBUK, Korea (Email : stefano@, kunsan.ac.kr)

Bong-Man Ahn is with the NURI, JEONBUK National Unversity, Korea

Jee-Wonl Hwang is with the Department of Information Technology Eng., JEONBUK National Unversity, JEONBUK, Korea, Corresponding author (Email : hwangj@jbnu.ac.kr) be obtained directly.

In this paper, we present an advanced Wiener-Hopf equation for producing coefficients of TDL filters directly in a lattice filter (or adaptation filter that uses the GramSchmidt orthogonalization process, etc.). In this paper, the new algorithm of the Wiener-Hopf equation will be referred to as an equivalent Wiener-Hopf equation.

A novel cost function is defined and applied to the analysis of the MSE(minimum mean square error) for the purpose of evaluating the performance of the proposed method. The results show that the MMSE values of both methods are theoretically same. Furthermore, a comparison between the proposed algorithm and the original Wiener-Hopf equation was made to determine the performance of the proposed algorithm by applying the method to the identification of an unknown system.

This experiment showed similar results for both methods. It can be concluded that the proposed method has the following contributions.

Firstly, it provides a simple method of finding the coefficient of a TDL (tapped-delay-line) filter in the case where a lattice filter or the process of Gram-Schmidt orthogonalization is used. Secondly, a new cost function is defined. This cost function may facilitate research in the adaptive signal processing area.

This paper is organized as follows. Section $I I$ proposes an equivalent Wiener-Hopf equation. Cost function and MMSE are discussed in Section III. The simulation results and conclusions are summarized in Sections III and IV, respectively.

\section{DERIVATION OF EQUIVALENT WIENER- HOPF EQUATION}

Fig. 1 illustrates the structure of an adaptive filter with an orthogonal input vector. In this figure, we can see the overall block diagram for this paper.

In Fig. 1, the input signal vector $\boldsymbol{x}(n)$, optimal coefficient vector $\boldsymbol{w}_{\text {opt }}$, orthogonal input vector $\boldsymbol{b}(n)$ and regression coefficient $\boldsymbol{k}$ are vectors. And $\boldsymbol{Q}$ is an $M \times M$ orthogonal transform matrix.

The signal $d(n)$ is the desired response, $\hat{d}(n)$ is the estimated desired response and $v(n)$ is the measurement noise. 


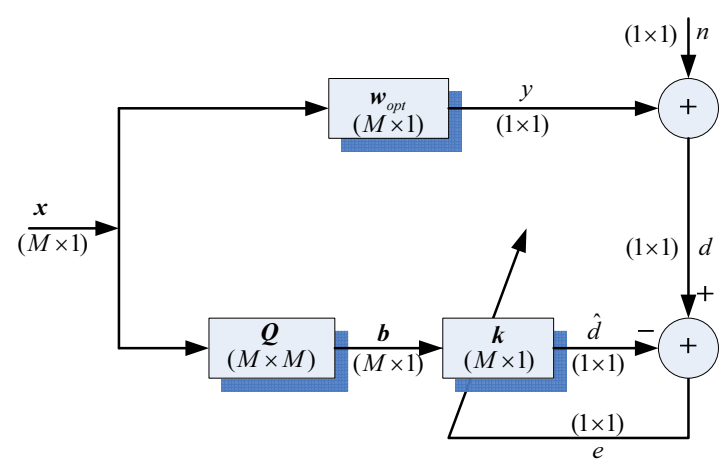

Fig. 1. Structure of an adaptive filter using an orthogonal input vector.

As shown in Fig. 1, the system is excited by an input signal $\boldsymbol{x}(n)$, and we are looking for the estimated values of the $M$, the unknown tap coefficients, so that the error $e(n)$ between the measured output $d(n)$ and the output of an associated model $\hat{d}(n)$ is minimum in terms of the least mean squared value.

That is,

$$
J=E\left[e(n) e^{*}(n)\right]=E\left[|e(n)|^{2}\right.
$$

is a minimum.

The error is defined as following

$$
e(n)=d(n)-\boldsymbol{k}^{H} \boldsymbol{b}(n)
$$

where, $(H)$ is a Hermitian transpose. $\hat{d}(n)$ and $\boldsymbol{b}(n)$ are expressed as follows

$$
\begin{aligned}
& \hat{d}(n)=\boldsymbol{k}^{H} \boldsymbol{b}(n)=\boldsymbol{k}^{H} \boldsymbol{Q x}(n) \\
& \boldsymbol{b}(n)=\boldsymbol{Q x}(n)
\end{aligned}
$$

And we define the desired response $d(n)$ as

$$
d(n)=\boldsymbol{w}_{\text {opt }}^{H} \boldsymbol{x}(n)
$$

The error $e(n)$ reaches zero if $d(n)$ is equal to $\hat{d}(n)$. That is,

$$
d(n)-\hat{d}(n)=\left[\boldsymbol{w}_{\text {opt }}^{H}-\boldsymbol{k}^{H} \boldsymbol{Q}\right] \boldsymbol{x}(n)=0
$$

If $\boldsymbol{x}(n) \neq 0$, the solution of the above equation becomes

$$
\boldsymbol{w}_{\text {opt }}=\boldsymbol{Q}^{H} \boldsymbol{k}
$$

This result is similar to that shown in reference [1]. Generally, if the orthogonal input vector is used, the regression coefficient $\boldsymbol{k}$ and the coefficient of the TDL filter $\boldsymbol{w}_{\text {opt }}$ are related to each other, as presented in (7). In this case, if the regression coefficient $\boldsymbol{k}$ is known, the transformation matrix $Q$ must be known in order to determine the $\boldsymbol{w}_{\text {opt }}$.

Using (1), the gradient of the mean-squared error can be obtained as following

$$
\frac{\partial J}{\partial \boldsymbol{k}}=2 E\left[\left(d(n)-\boldsymbol{k}^{H} \boldsymbol{b}(n)\right)\left(-\boldsymbol{b}^{H}(n)\right)\right]
$$

From the above equation, the vector $\boldsymbol{k}$ can be represented as

$$
\boldsymbol{k}=E\left[\boldsymbol{b}(n) \boldsymbol{b}^{H}(n)\right]^{-1} E\left[\boldsymbol{b}(n) d^{*}(n)\right]
$$

Rewriting the above equation using a matrix form, we obtain.

$$
\begin{aligned}
\boldsymbol{k} & =\boldsymbol{R}_{b b}^{-1} \boldsymbol{p}_{b d} \\
\boldsymbol{R}_{b b} & =E\left[\boldsymbol{b}(n) \boldsymbol{b}^{H}(n)\right] \\
\boldsymbol{p}_{b d} & =E\left[\boldsymbol{b}(n) d^{*}(n)\right]
\end{aligned}
$$

Where $\boldsymbol{R}_{b b}$ is an autocorrelation matrix of $\boldsymbol{b}(n)$, We have to assume that $\boldsymbol{R}_{b b}$ is a nonsingular matrix. The vector $\boldsymbol{p}_{b d}$ is a cross-correlation vector between the desired response and the orthogonal signals. The equation (10) can be used to obtain the regression coefficient vector if the orthogonal input signal is applied. In the conventional method, the regression coefficient $\boldsymbol{k}$ can be calculated by using (9) or (10) and then the coefficient of the TDL filter $\boldsymbol{w}_{\text {opt }}$ is determined by using (7).

In this paper, we proposed the theorem that can determine $\boldsymbol{w}_{\text {opt }}$ directly without any calculation of $\boldsymbol{k}$.

Theorem 1. If the desired response $d(n)$ and the estimated desired response $\hat{d}(n)$ have so similar values, the relationship $\boldsymbol{R}_{b x} \boldsymbol{w}_{\text {opt }}=\boldsymbol{p}_{b d}$ is defined.

proof)

Substituting (4) and (7) into (8), we get

$$
\frac{\partial J}{\partial \boldsymbol{k}}=2 E\left[\left(d(n)-\boldsymbol{w}_{\text {opt }}^{H} \boldsymbol{Q}^{-1} \boldsymbol{Q} \boldsymbol{x}(n)\right)\left(-\boldsymbol{b}^{H}(n)\right)\right]
$$

The optimal coefficient $\boldsymbol{w}_{\text {opt }}$ can be obtained from (13) using $\boldsymbol{Q}^{-1} \boldsymbol{Q}=I$.

$$
E\left[\boldsymbol{b}(n) \boldsymbol{x}^{H}(n)\right] \boldsymbol{w}_{\text {opt }}=E\left[\boldsymbol{b}(n) d^{*}(n)\right]
$$

The equation (14) can be simplified as

$$
\boldsymbol{R}_{b x} \boldsymbol{w}_{\text {opt }}=\boldsymbol{p}_{b d}
$$

where

$$
\boldsymbol{R}_{b x}=E\left[\boldsymbol{b}(n) \boldsymbol{x}^{H}(n)\right]
$$

The new version (15) of the Wiener-Hopf equation will be referred to herein as an equivalent Wiener-Hopf equation.

The matrix $\boldsymbol{R}_{b x}$ in (16) is the correlation matrix of the orthogonal input vector $\boldsymbol{b}(n)$ and nonorthogonal input vector $\boldsymbol{x}(n)$, and $\boldsymbol{R}_{b x}$ is nonsingular[1]. Also, the matrix $\boldsymbol{R}_{b x}$ is an upper triangular matrix having a nonToeplitz structure. The (15) is the result of the partial 
differentiation of the cost function $J($.$) with respect to$ $\boldsymbol{k}$, as shown in (10). This indicates that we can obtain $\boldsymbol{w}_{\text {opt }}$ while keeping the physical meaning of (12). Therefore, the coefficient $\boldsymbol{w}_{\text {opt }}$ of the TDL filter can be determined easily from the filter of the input $\boldsymbol{b}(n)$ that has been orthogonally transformed with respect to the input $\boldsymbol{x}(n)$, if (14) is used.

The algorithm based on (10) is used to develop a new algorithm, since the matrix $\boldsymbol{R}_{b b}$ is a diagonal matrix and it is easy to obtain its inverse matrix [7].

It has been used mainly in lattice filters, etc. However, it requires the additional computation of transformation by means of a suitable process, since $\boldsymbol{k}$ is not a TDL coefficient. However, the problems mentioned above can be solved easily if (15) is used.

\section{ANALYSIS OF MINIMUM MEAN SQUARE ERROR}

The following equation can be derived from (13)

$$
\frac{\partial J}{\partial \boldsymbol{k}}=2 E\left[\left(d(n)-\boldsymbol{w}_{\text {opt }}^{H} \boldsymbol{x}(n)\right)\left(-\boldsymbol{b}^{H}(n)\right)\right]
$$

Using (17), the following equation can be determined easily

$$
J=E\left[\left(d(n)-\boldsymbol{w}_{\text {opt }}^{H} \boldsymbol{x}(n)\right)\left(d^{*}(n)-\boldsymbol{b}^{H}(n) \boldsymbol{k}\right)\right]
$$

It is reasonable to use the cost function shown in (18) since the error spaces created from $\boldsymbol{w}_{\text {opt }}$ and $\boldsymbol{k}$ are generally the same [1].

From (18), we get

$$
\begin{aligned}
J= & E\left[d(n) d^{*}(n)\right]-E\left[d(n) \boldsymbol{b}^{H}(n)\right] \boldsymbol{k} \\
& -\boldsymbol{w}_{\text {opt }}^{H} E\left[\boldsymbol{x}(n) d^{*}(n)\right]+\boldsymbol{w}_{\text {opt }}^{H} E\left[\boldsymbol{x}(n) b^{H}(n)\right] \boldsymbol{k}
\end{aligned}
$$

Let's represent $\boldsymbol{w}_{\text {opt }}$ without $\boldsymbol{k}$ since (19) includes both $\boldsymbol{w}_{\text {opt }}$ and $\boldsymbol{k}$.

Substituting $\boldsymbol{w}_{\text {opt }}$ of (15) into (19), we get

$$
\begin{aligned}
J & =E\left[d(n) d^{*}(n)\right]-\boldsymbol{p}_{b d}^{H} \boldsymbol{k}-\boldsymbol{p}_{b d}^{H} \boldsymbol{R}_{b x}^{-H} \boldsymbol{p}_{x d}+\boldsymbol{p}_{b d}^{H} \boldsymbol{R}_{b x}^{-H} \boldsymbol{R}_{b x}^{H} \boldsymbol{k} \\
& =E\left[d(n) d^{*}(n)\right]-\boldsymbol{p}_{b d}^{H} \boldsymbol{R}_{b x}^{-H} \boldsymbol{p}_{x d} \\
& =E\left[d(n) d^{*}(n)\right]-\boldsymbol{w}_{o p t}^{H} \boldsymbol{p}_{x d}
\end{aligned}
$$

In (20), the MMSE(minimum mean square) value is represented. And (21) represents the same results as those in the original Wiener-Hopf equation.

$$
J_{\min }=\sigma_{d}^{2}-\boldsymbol{w}_{\text {opt }}^{H}(n) \boldsymbol{p}_{x d}
$$

Where $\sigma_{d}^{2}=E\left[d^{2}(n)\right]$.

\section{COMPUTER SIMULATION}

To evaluate the performance of the proposed algorithm, the identification of an unknown FIR system is performed. The input signal $x(n)$ to the adaptive filter was obtained from the output of a low pass filter which transfer function is as follows;

$$
H(z)=\frac{1}{1-0.98 z^{-1}+0.693 z^{-2}-0.22 z^{-3}+0.309 z^{-4}-0.177 z^{-5}}
$$

where, its input is a white, zero mean and pseudorandom Gaussian noise. The response signal $y(n)$ was obtained from the FIR system, as shown in (23).

$$
H(z)=2.65-3.31 z^{-1}+2.24 z^{-2}-0.7 z^{-3}
$$

The desired response was obtained by adding some measurement noise that has a suitable $\mathrm{S} / \mathrm{N}$ ratio to the output of (23). The measurement noise signal was an additive white, zero mean and pseudorandom Gaussian and was uncorrelated with the input signal.

The computer simulation is designed to determine the similarities of the coefficient of the filters by solving the Wiener-Hopf equation and the equivalent Wiener-Hopf equation. All results presented in this paper are ensemble averages of 100 independent runs with 9000 data.

Fig. 2 shows that the curves of the $\left(\boldsymbol{w}_{x x}^{(i)}-\boldsymbol{w}_{\text {opt }}^{(i)}\right) / \boldsymbol{w}_{\text {opt }}^{(i)}$, $\left(\boldsymbol{w}_{b x}^{(i)}-\boldsymbol{w}_{o p t}^{(i)}\right) / \boldsymbol{w}_{o p t}^{(i)}$ and $\left(\boldsymbol{w}_{b s}^{(i)}-\boldsymbol{w}_{o p t}^{(i)}\right) / \boldsymbol{w}_{o p t}^{(i)}$ obtained from the solutions of the Wiener-Hopf and equivalent Wiener-Hopf equation for an SNR of $-90 \mathrm{~dB},-30 \mathrm{~dB}$, $10 \mathrm{~dB}$ and $10 \mathrm{~dB}$, respectively. $\boldsymbol{w}_{\text {opt }}$ is the i-th optimal coefficient, $\boldsymbol{w}_{x x}$ is the $\mathrm{i}$-th solution of original WienerHopf equation and $\boldsymbol{w}_{b x}^{(i)}$ is the i-th solution of the proposed equivalent Wiener-Hopf equation. The $\boldsymbol{R}_{b s}$ are assumed to be triangular matrix. The back substitution method was applied to $\boldsymbol{R}_{b s}$ obtain the coefficients $\boldsymbol{w}_{b s}^{(i)}$.

Fig. 2 presents that the difference between the solution of the Wiener-Hopf equation and the equivalent WienerHopf equation is very minor under the SNR $=-90 \mathrm{~dB}$, $30 \mathrm{~dB},-10 \mathrm{~dB}$ and $10 \mathrm{~dB}$.

From these results, it is possible to conclude that two solutions of Wiener-Hopf equation and the equivalent Wiener-Hopf equation are very similar.

Also, it is shown that the optimal coefficient can be obtained by using the proposed method. From Fig. 2, it is possible to determine that the difference between the coefficient and the optimal coefficient obtained using back substitution is constant, regardless of the noise. But this method shows poor performance compared to other schemes.

The following simulation is made when the rank is deficient. Fig. 3 illustrates the results obtained by varying 
the rank of autocorrelation. In Fig. 3, we set an order of unknown system to 20 and one of computer simulation to 10 under SNR of $-30 \mathrm{~dB}$, respectively.

Fig. 3 shows the results of the coefficients for various ranks of the correlation matrix. The matrix is full rank. The figure shows that both the existing method and the proposed method have similar performance. Fig. 3 (b) is the case of a rank deficiency.
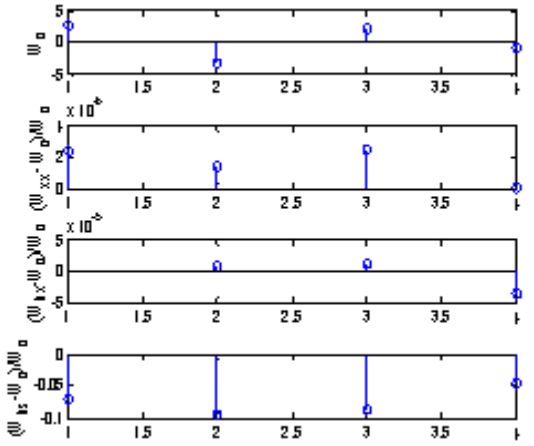

(a)
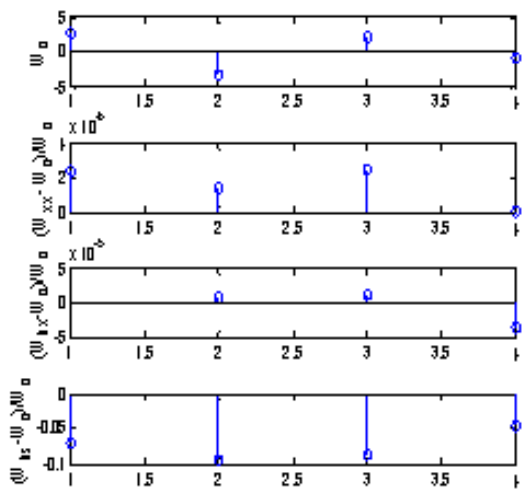

(b)
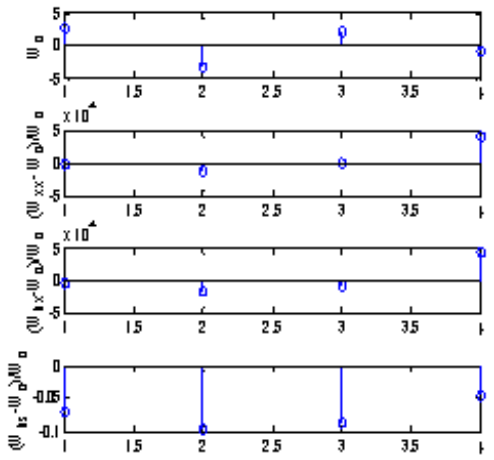

(c)
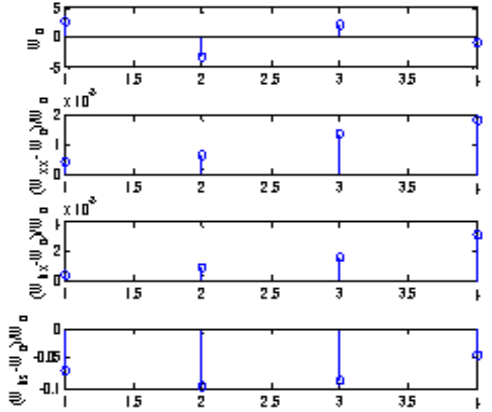

(d)

Fig. 2. Comparison of coefficients with various $\mathrm{S} / \mathrm{N}$ ratios.
(a) $\mathrm{S} / \mathrm{N}=-90 \mathrm{~dB}$ (b) $\mathrm{S} / \mathrm{N}=-30 \mathrm{~dB}$ (c) $\mathrm{S} / \mathrm{N}=-10 \mathrm{~dB}$

(d) $\mathrm{S} / \mathrm{N}=10 \mathrm{~dB}$

This figure shows the simulation results of an unknown system of order 20, while the order of the simulated adaptive filter is 10 . The figure shows that the proposed method and existing method produce similar results.

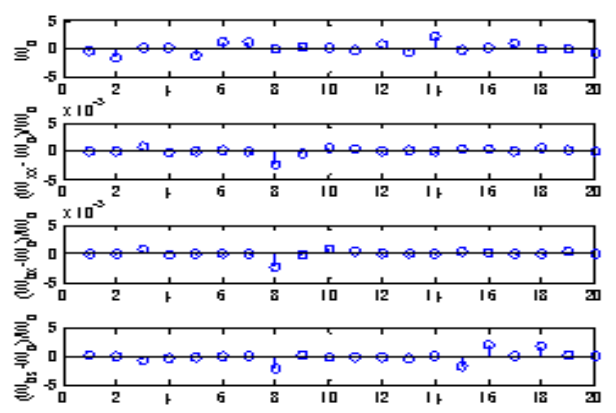

(a)

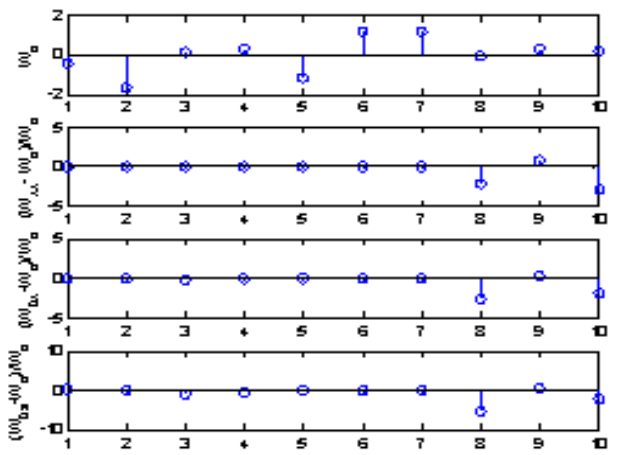

(b)

Fig. 3. Comparison of coefficients according to variation of correlation matrix.

(a) order of unknown system; 20, order of system identification; 20.

(b) order of unknown system;20, order of system identification; 10 . 
Consequently, the various experiments showed that the solution of the proposed method is as good as that of the original Wiener-Hopf equation.

\section{CONCLUSIONS}

In this paper, an equivalent Wiener-Hopf equation is proposed. The proposed algorithm can determine the coefficient of a TDL filter directly when the adaptive filter has an orthogonal input signal. Also, a theoretical analysis was performed for the MMSE using a solution to the equivalent Wiener-Hopf equation. Furthermore, the proposed algorithm shows similar performance to that of the original Wiener-Hopf equation.

The proposed algorithm has the advantage of allowing the application area of adaptation filters which have an orthogonal input signal to be expanded. This is because the performance of the proposed algorithm is similar to that of the Wiener-Hopf equation. For the validation of the proposed algorithm, the original Wiener-Hopf equation and the proposed algorithm were applied to an unknown system identification problem. The results showed that the proposed method produces the optimal coefficient vectors as in the case of the Wiener-Hopf equation.

\section{REFERENCES}

[1] Haykin, Adaptive Filter Theory - Fourth Edition, Prentice Hall.

[2] J. G. Proakis, C. M. Rader, F. Ling and C. L. Nikias, Advanced Digital Signal Processing. Macmillan Publishing Company, 1992.

[3] P. S. R. Diniz, Adaptive Filtering-Algorithms and Practical Implementation, second edition. Kluwer Academic Publishers, 2002.

[4] J. Makhoul. "A Class of ALL-Zero Lattice Digital Filters: Properties and Applications," IEEE Trans. Acoustics, Speech, and Signal Processing, vol. ASSP-26, pp.304-314, Aug. 1978.

[5] E. Karlsson, M. Hayes, "Least squares ARMA modeling of linear time-varying systems: Lattice filter structures and fast RLS algorithms," IEEE Trans. Acoustics, Speech, and Signal Processing, vol. ASSP-35, NO. 7, pp. 994 - 1014, July 1987.

[6] H. K. Baik, V. J. Mathews, "Adaptive lattice bilinear filters," IEEE Trans. on Signal Processing, vol. 41, pp. 2033 - 2046 , June 1993.

[7] L. Fuyun, J. Proakis, "A generalized multichannel least squares lattice algorithm based on sequential processing stages," IEEE Trans. on Signal Processing, vol.32, pp. 381 - 389, Apr. 1984

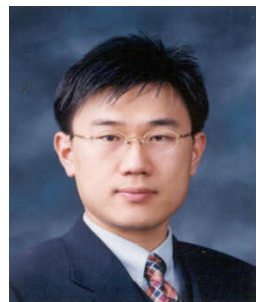

Ju-Phil Cho received the M.S. and $\mathrm{PhD}$ degrees in Electronics Engineering from Jeonbuk National University in Jeonju, Korea, in 1994 and 2001 respectively, From 2000 to 2005, he was a Senior Research Staff at ETRI, Taejeon, Korea, where he was involved on the development of a code division multiple access (CDMA) digital cellular system, 4G cellular system, Cognitive Radio. Since 2005, he has been an associate professor at Department of Radio-communciation Engineering, Kunsan National University, Kunsan, Korea. His current research interest is the wireless communications technology including spectrum sensing, WiBro, LTE, and Cognitive Radio.

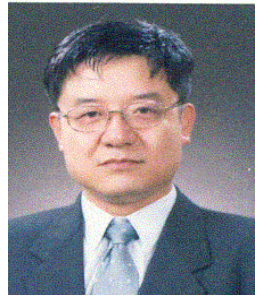

Bong-Man Ahn received the B.S., M.S. and $\mathrm{PhD}$ degrees in Electronics Engineering from Jeonbuk National University in Jeonju, Korea, in 1988, 1990 and 1997 respectively, From 1995 to 2004, he was a professor at Jeonbuk Science College, Jeongup, Korea. Since 2006, he has been an researcher at Next Group in Jeonbuk National University, Jeonju, Korea. His current research interest is the nonlinear signal processing, high-speed adaptive filter and wireless communications technology.

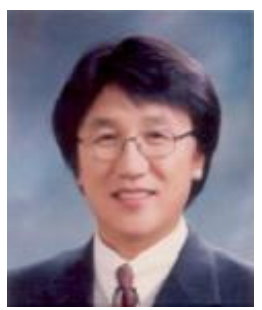

Jee-Won Hwang received the B.S., M.S. and $\mathrm{PhD}$ degrees in Electronics Engineering from Jeonbuk National University in Jeonju, Korea, in 1985, 1987 and 1995 respectively, From 1992 to 2007 , he was a professor at Iksan National University, Iksan, Korea. Since 2008, he has been a professor at Department of Information Technology in Jeonbuk National University, Jeonju, Korea. His current research interest is computer architecture, the nonlinear signal processing, high-speed adaptive filter and wireless communications technology. 